\title{
Towards an Integrated Information Logistics for Multi Hazard Early Warning Systems
}

\author{
Matthias Lendholt* and Martin Hammitzsch
}

GFZ German Research Centre for Geosciences, Germany

\begin{abstract}
The delivery of user-tailored warning messages for heterogeneous user groups is a challenge rarely covered by hazard monitoring and Early Warning Systems (EWS) even though it is one of the four key elements of early warning systems identified by the United Nations International Strategy for Disaster Reduction (UN/ISDR). This article describes the concepts of generic information logistics developed for the distant early warning system (DEWS). It is not limited to specific hazard types, languages or other deployment specifics. Instead, it enables the generation of user-tailored warning messages that account for specific needs, individual requirements, different levels of understanding, distinct perceptions and varying personal abilities and it provides several filter mechanisms to avoid unintentional message flooding in emergency situations. This novel approach is completed by a reusable graphical user interface component, which provides functionality to generate warning messages in compliance with the Common Alerting Protocol (CAP) standard to leverage inter-operability among early warning systems.
\end{abstract}

Keywords: Disaster management, early warning, alert system, information logistics, CAP.

\section{INTRODUCTION}

The United Nations International Strategy for Disaster Reduction (UN/ISDR) have identified four inter-related key elements of effective Early Warning Systems (EWS): (1) risk knowledge, (2) monitoring and warning service, (3) dissemination and communication, and (4) response capability [1]. This definition covers all capacities needed to build an efficient infrastructure for generation and dissemination of meaningful warning information of possible extreme events or disasters that threaten people's lives. This includes the tasks of data collection and risk assessment - both belonging to the first item risk knowledge - as well as capacity building, which belongs to the fourth element - response capability. These long-lasting tasks have to be continuously carried out to achieve an overall preparedness for emergencies, to act appropriately to reduce the possibility of harm or loss.

Focussing on the technical infrastructure of the EWS, another definition outlines its main tasks: the basic functionality of EWS consists of (1) detecting the event and (2) quickly warning the public [2]. Whilst the first topic has been covered by several projects and productive EWS, the second is still often disregarded; even though it is of utmost importance and weakness in one element can result in the failure of the entire system. The detection of hazards (monitoring) is based on the upstream information flow from physical processes to the desktop early warning application, where hazard specific data is displayed and aggregated with

*Address correspondence to this author at the German Research Centre for Geosciences Germany: Tel: +49 331288 1687; Fax: +49 331288 1703;

E-mail: matthias.lendholt@gfz-potsdam.de

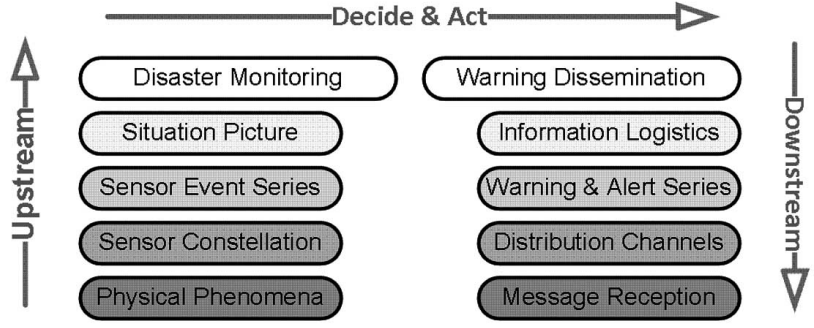

Fig. (1). Upstream and downstream event and information flow in early warning systems

relevant information ("Upstream" in Fig. 1) to generate the situation picture for operators. They are in charge of situation evaluation and decision making ("Decide \& Act" in Fig. 1).

The downstream information flow (UN/ISDR: dissemination and communication), including the generation of warning products with evacuation orders or the activation of disaster control procedures, is frequently beyond the scope of EWS and research projects. These procedures are carried out by local administration authorities in the military or civil protection infrastructure. The interface between both worlds often consists of person to person interactions, such as regular status reports or simple phone calls, in emergency situations. This modus operandi designates a person in charge who has to justify their actions and who is ultimately responsible for making critical decisions such as evacuation orders. However, this modus operandi is not suited for all situations and hazards. The time critical nature of several types of man-made and natural hazards, such as tsunamis or 
made and natural hazards, such as tsunamis or nuclear meltdowns, is an elementary part of the disaster and its progression. Another dimension is the spatial expansion of the disaster; if the threat exceeds a certain threshold, it is no longer possible to adhere to the manual procedures described above. The overabundance of data, combined with a high degree of urgency, rules out the possibility of in-time early warning because human interactions might not be fast enough to inform all affected subjects such as civilians, rescue services, civil protection agencies or military forces. Finally, information demand varies among different user groups. Thus, information logistics applications must generate individual messages to serve different needs [3]. The challenge is to reach only the appropriate recipients as efficiently as possible [4]. In the event of a disaster, the automatic processing of available data, and forecasts, in combination with message generation and dissemination, must be realised by information logistics that seamlessly glue together both the upstream data flow and the message dissemination parts of an early warning centre infrastructure. The concepts presented in this paper to counter these challenges incorporate the requirements and specifications of the UNESCO Intergovernmental Oceanic Commission (IOC) for the development of oceanwide tsunami EWS as described in [30]. Although the presented information logistics are not limited to tsunami hazards, achieving compliance with IOC specifications was a main objective of the Distant Early Warning System (DEWS) project, which is described below and in which context this research took place. Furthermore, the developed concepts incorporate and are verified against the demands listed in the UN/ISDR checklist for EWS [1].

\section{RELATED WORK}

The development of EWS for both man-made and natural hazards is the focus of various research projects covering a wide range of topics, including the following:

- Analysis of geophysical events and their effects

○ Development of in-field sensor technologies and their applicability

- Simulation-based risk assessment for public infrastructure and industrial facilities

- Development and utilisation of international standards, such as the integration of heterogeneous sensor systems using OGC (Open Geospatial Consortium) Sensor Web Enablement (SWE) standards

While most attention has focused on sensor measurements and disaster prediction, the delivery of user tailored warning messages for heterogeneous user groups is a challenge rarely covered by hazard monitoring and EWS. Instead, warning messages are often based on administrative or technical terminology and are addressed to specialists, but are not intended to inform the general public. Over the past few years, several early warning projects have attempted to tackle this issue. This chapter depicts and outlines the most relevant projects and their activities in this field. Though the developed concepts possess similar strategies, to date, an overall generic concept for EWS is missing.
The Weather Information on Demand service (WIND) provides information about adverse weather conditions based on user needs with respect to content, location, time and quality [3]. This project was a driving force in the development of information logistics applications in EWS, and several ideas have been incorporated and enhanced in this work. In CHORIST (Integrating Communications for en Hanced envir Onmental RISk management and citizens safety), a research project of the $6^{\text {th }}$ Framework Programme (FP) of the European Commission (EC), a warning subsystem was designed and developed to provide a template-based Message Creator \& Dispatcher component that is connected to dissemination channel gateways [2]. All warning products are manually qualified, selected and created by human operators, and are thus limited for urgent warnings dealing with high volumes of data. ERMA, (Electronic Risk Management Architecture for Small- and Medium-sized communities), also a $6^{\text {th }}$ FP project, outlines a citizen relationship management system to support communication with citizens [5]. It is focused on local communities and provides a collaboration platform; but it is limited in addressing the requirements of large-scale EWS in tackling large spatial areas and multilingualism. In GITEWS (German Indonesian Tsunami Early Warning System), a Decision Support System (DSS) generates tsunami warning messages based on templates. Different message types and languages are supported, as well as the subsequent insertion of generated warning messages into Common Alerting Protocol (CAP) container files [6]. The Istanbul earthquake rapid response and early warning system (IERREWS) provides notifications for infrastructure facilities (e.g., metro, gas and electricity distribution networks, industrial facilities). The earthquake early warning signals will be transmitted to the end users by employing communication companies as service providers [7]. These notifications are suited for automated emergency shut-downs in the event of strong and non-ambiguously measurable earthquakes, and are thus less appropriate as a public EWS that covers a wide range of user groups. The alpEWAS (Early Warning System for Alpine Slopes) project, dealing with landslide monitoring and early warning, offers basic email alarming and notification functions [8]. Technical messages that track sensor plug-in statuses are automatically sent to administrators, and notification of sensor data can be activated using thresholds for any of the handled datasets, such as deformation rate. Because no pre-processing or forecasting assesses the impact of these measurements, the message content is, according to the "data, information, knowledge, wisdom" (DKIW) hierarchy [9], on the data-level and is thus inappropriate for most target groups except scientists or early warning experts. Moreover, multiple web based information systems or EWS provide notifications on hazards like earthquakes, tsunamis, hurricanes or volcanic eruptions. However, they are all limited in one or more dimensions regarding spatial reference, message filtering, multilingualism, dissemination channels, preciseness or accuracy and are often focused on a certain target audience.

\section{FRAMING CONDITIONS}

Developing the information logistics was inspired by official recommendations of UN ISDR and UN IOC, however, other framing conditions were also taken into account such 
as literature and theory dealing with human responses to warnings. In addition, best practices, technical standards and drawbacks of existing EWS were analysed and accounted.

\section{REQUIREMENTS FOR CONSTRUCTING WARNING MESSAGES}

One theory dealing with human response to warnings for natural hazards is the Protective Action Decision Model (PADM) by Lindell and Perry. It explains people's protective action decision in response to imminent disasters [10, 11]. As a conclusion of their research, Lindell and Perry created a list of recommendations for constructing warning messages. "Warning messages should be specific and as brief as possible, so that citizens will not to have to go through irrelevant information that increases the likelihood of distraction" [12]. Warning messages should answer the following questions [11]:

1. Who is issuing the warning?

2. What type of event is threatening?

3. Who is being threatened?

4. When is the anticipated impact to occur at recipient's location?

5. How intense is the event expected to be at the warning recipient's location?

6. How probable is that the event will strike the warning recipient's location?

7. What specific protective actions should be taken?

8. Are there high risk groups that require special actions?

\section{UN/ISDR CHECKLIST FOR EARLY WARNING SYSTEMS}

On the third International Conference on Early Warning, held March 2006 in Bonn, Germany, a checklist for EWS was published. "The checklist, which is structured around the [aforementioned] key elements of effective EWS, aims to be a simple list of the main elements and actions that national governments or community organizations can refer to when developing or evaluating EWS; or simply to check that crucial procedures are in place" [1]. However, even the checklist addresses the four elements of people-centred EWS, the checklist focuses on organizational issues, established processes and the overall disaster management cycle. This includes organizational arrangements, identification of natural hazards, risk assessments, et cetera. Only a subset of all checklist items addresses the systems and their components of early warning centres. The following list selects the relevant checklist items for the presented information logistics. In particular, key element 3 "Dissemination and communication" is of relevance. The identified items were taken up again during system validation (Table 2). The reference ID establishes traceability between Table $\mathbf{1}$ and Table $\mathbf{2}$.

\section{REQUIREMENTS OF GENERIC INFORMATION LOGISTICS}

Based on the requirements for warning messages, and the incorporated UN requirements, fundamental prerequisites for generic information logistics become apparent. A generic reference architecture for multi hazard EWS should have components designed to serve in new deployments and new sites without re-programming or compilation procedures. Instead, deployment-specific add-ons or plug-ins should be easily added based on the needs of a specific scenario. Consequently, not only must the upstream be unbound to specific hazard characteristics, but also the downstream, including the warning message generation and its dissemination, must be realised independent of any hazard and infrastructure specific characteristics. Therefore, the information logistics must not be hazard-specific and must not be limited to a predefined set of message types. Hazard-specific message types, such as tsunami warnings, must instead be dynamically addable. The information logistics must also be independent from any specific dissemination channel. An early warning centre must support a wide range of dissemination channels, such as text messaging, email, fax, and twitter, but must also provide products for TV and radio stations. Moreover, warning products must contain an adequate common situational picture and, if required, clear and understandable instructions. User needs must be respected to ensure and enable proper message reception for all recipients. Further key requirements:

- Message notification strategies / message filtering: Different user groups are interested in different message types. For example, rescue services are interested in alert status changes in order to begin preparations according to crises that might result from an ongoing threat. In contrast, civilians must not be confused with low priority or highly detailed messages because these could reduce the importance of the received warning messages and false alarms result in lower risk awareness and a general loss of public confidence in warning systems [13].

- Area of interest: Only message products that spatially reference the area of interest should be delivered to recipients. Spatial referencing has to distinguish between users in a static area of interest, such as rescue services responsible for a region that spans one or more political subdivisions and mobile users such as civilians who are interested in warning messages for the area they are currently staying in. This second category must be reachable by dissemination channels that establish the spatial reference themselves. For example, Cell Broadcasting is considered a viable alternative to text messaging, as it uses a common broadcast channel to disseminate text messages to all subscribers at once within a defined geographical broadcast area [14].

- Message vocabulary: The vocabulary of how information is phrased and what degree of detail is used are of high importance in critical emergency situations. Of all the requirements that a public warning must fulfil, being understandable may be the most crucial for the warning's overall success [15]. Depending on the message receiver's role in a hazard, a specific message vocabulary is crucial to ensuring proper message reception. Civilians need unambiguous event descriptions, together with clear 
Table 1. Applicability of UN/ISDR Checklist Items [1]

\begin{tabular}{|c|c|c|}
\hline Checklist Item & Applicability & Ref. ID \\
\hline \multicolumn{3}{|l|}{ Cross Cutting Issues } \\
\hline 1 Effective Governance & Organizational aspect, not applicable. & \\
\hline 2 A Multi-Hazard Approach & Functional requirement. & 1 \\
\hline 3 Involvement of Local Communities & Organizational aspect, not applicable. & \\
\hline 4 Gender Perspectives and Cultural Diversity & Relevant for warning message definition. & 2 \\
\hline \multicolumn{3}{|l|}{ Key element 1: Risk Knowledge } \\
\hline [All items] & $\begin{array}{l}\text { Not applicable: The items address organizational and procedural } \\
\text { issues or deal with different disaster management issues. }\end{array}$ & \\
\hline \multicolumn{3}{|l|}{ Key element 2: Monitoring and Warning Service } \\
\hline $\begin{array}{l}\text { 1.2 Agreements and interagency protocols established to ensure } \\
\text { consistency of warning language }[\ldots]\end{array}$ & Organizational aspect but also functional requirement. & 3 \\
\hline $\begin{array}{l}1.5 \text { Protocols in place to define communication responsibilities and } \\
\text { channels for technical warning services }\end{array}$ & $\begin{array}{l}\text { Organizational aspect but also request to support technical warning } \\
\text { services (non-human interaction). }\end{array}$ & 4 \\
\hline $\begin{array}{l}\text { 3.2 Data and warning products issued within international standards } \\
\text { and protocols }\end{array}$ & Functional requirement. & 5 \\
\hline $\begin{array}{l}\text { 3.6 Warnings generated and disseminated in an efficient and timely } \\
\text { manner and in a format suited to user needs }\end{array}$ & $\begin{array}{l}\text { The first part is a non-functional requirement ("timely manner"). } \\
\text { The second part a functional requirement. }\end{array}$ & 6 \\
\hline [All other items] & $\begin{array}{l}\text { Not applicable: The items address institutional and procedural is- } \\
\text { sues. }\end{array}$ & \\
\hline \multicolumn{3}{|l|}{ Key element 3: Dissemination and Communication } \\
\hline $\begin{array}{l}1.1-1.5 \text { [All items of (1) "Organizational and Decision-making } \\
\text { Processes Institutionalized") }\end{array}$ & Not applicable: Addressing organizational and procedural aspects. & \\
\hline $\begin{array}{l}\text { 2.1 Communication and dissemination systems tailored to the needs } \\
\text { of individual communities }\end{array}$ & $\begin{array}{l}\text { Deployment specific requirement but also requirement regarding } \\
\text { information logistics capabilities. }\end{array}$ & 7 \\
\hline $\begin{array}{l}\text { 2.2. Warning communication technology reaches the entire popula- } \\
\text { tion, including seasonal populations and remote locations. }\end{array}$ & Deployment specific requirement/check. Not applicable. & \\
\hline $\begin{array}{l}\text { 2.3 International organizations or experts consulted to assist with } \\
\text { identification and procurement of appropriate equipment. }\end{array}$ & $\begin{array}{l}\text { Deployment and organisation specific requirement/check. Not ap- } \\
\text { plicable. }\end{array}$ & \\
\hline $\begin{array}{l}\text { 2.4 Multiple communication mediums used for warning dissemina- } \\
\text { tion (e.g. mass media and informal communication). }\end{array}$ & Functional requirement. & 8 \\
\hline $\begin{array}{l}2.5 \text { Agreements developed to utilise private sector resources where } \\
\text { appropriate (e.g. amateur radios, safety shelters). }\end{array}$ & Deployment specific check. Not applicable. & \\
\hline $\begin{array}{l}\text { 2.6 Consistent warning dissemination and communication systems } \\
\text { used for all hazards. }\end{array}$ & Requirement regarding multi hazard functionality & 9 \\
\hline $\begin{array}{l}2.7 \text { Communication system is two-way and interactive to allow for } \\
\text { verification that warnings have been received. }\end{array}$ & Functional requirement. & 10 \\
\hline $\begin{array}{l}\text { 2.8 Equipment maintenance and upgrade programme implemented } \\
\text { and redundancies enforced so back-up systems are in place in the } \\
\text { event of a failure. }\end{array}$ & Deployment specific check. Not applicable. & \\
\hline $\begin{array}{l}\text { 3.1 Warning alerts and messages tailored to the specific needs of } \\
\text { those at risk (e.g. for diverse cultural, social, gender, linguistic and } \\
\text { educational backgrounds). }\end{array}$ & Functional requirement & 11 \\
\hline $\begin{array}{l}3.2 \text { Warning alerts and messages are geographically-specific to } \\
\text { ensure warnings are targeted to those at risk only. }\end{array}$ & Functional requirement & 12 \\
\hline $\begin{array}{l}\text { 3.3 Messages incorporate the understanding of the values, concerns } \\
\text { and interests of those who will need to take action (e.g. instructions } \\
\text { for safeguarding livestock and pets). }\end{array}$ & Functional requirement & 13 \\
\hline $\begin{array}{l}\text { 3.4 Warning alerts clearly recognisable and consistent over time and } \\
\text { include follow-up actions when required. }\end{array}$ & Functional requirement & 14 \\
\hline 3.5 Warnings specific about the nature of the threat and its impacts. & Functional requirement & 15 \\
\hline
\end{tabular}


Table 1. cont...

\begin{tabular}{|c|c|c|}
\hline Checklist Item & Applicability & Ref. ID \\
\hline $\begin{array}{l}3.6 \text { Mechanisms in place to inform the community when the threat } \\
\text { has ended. }\end{array}$ & Functional requirement & 16 \\
\hline $\begin{array}{l}\text { 3.7 Study into how people access and interpret early warning mes- } \\
\text { sages undertaken and lessons learnt incorporated into message for- } \\
\text { mats and dissemination processes. }\end{array}$ & $\begin{array}{l}\text { Cultural aspect. Deployment specific / depending on geographic } \\
\text { area. Not applicable. }\end{array}$ & \\
\hline \multicolumn{3}{|l|}{ Key element 4: Response Capability } \\
\hline [All items] & $\begin{array}{l}\text { Not applicable: The items address sociological and organizational } \\
\text { aspects }\end{array}$ & \\
\hline \multicolumn{3}{|l|}{ Governance and Institutional Arrangements } \\
\hline [All items] & $\begin{array}{l}\text { Not applicable: The items address governmental and institutional } \\
\text { aspects }\end{array}$ & \\
\hline
\end{tabular}

Table 2. Validation Against UN/ISDR Checklist for Early Warning Systems

\begin{tabular}{|c|c|c|}
\hline Ref ID & Checklist Item & Validation \\
\hline 1 & 2 A Multi-Hazard Approach & $\begin{array}{l}\text { The information logistics has been designed hazard-type independently } \\
\text { and can be used in early warning systems for any natural hazard. }\end{array}$ \\
\hline 2 & 4 Gender Perspectives and Cultural Diversity & $\begin{array}{l}\text { The concept of message templates allows a deployment-specific defini- } \\
\text { tion of message types and message content that respects cultural diver- } \\
\text { sity of different geographic regions. }\end{array}$ \\
\hline 3 & $\begin{array}{l}\text { 1.2 Agreements and interagency protocols established to ensure } \\
\text { consistency of warning language }[\ldots]\end{array}$ & $\begin{array}{l}\text { The usage of standard protocols such as CAP and EDXL ensures inter- } \\
\text { agency operability. }\end{array}$ \\
\hline 4 & $\begin{array}{l}\text { 1.5 Protocols in place to define communication responsibilities and } \\
\text { channels for technical warning services }\end{array}$ & The presented concepts are not limited to a specific channel. \\
\hline 5 & $\begin{array}{l}\text { 3.2 Data and warning products issued within international standards } \\
\text { and protocols }\end{array}$ & International standards (CAP, EDXL) are used. \\
\hline 6 & $\begin{array}{l}\text { 3.6 Warnings generated and disseminated in an efficient and timely } \\
\text { manner and in a format suited to user needs }\end{array}$ & $\begin{array}{l}\text { The presented model leverages the usage of user-tailored warning mes- } \\
\text { sages respecting user's needs. }\end{array}$ \\
\hline 7 & $\begin{array}{l}\text { 2.1 Communication and dissemination systems tailored to the needs } \\
\text { of individual communities }\end{array}$ & $\begin{array}{l}\text { The ILC and the IDC support the parallel usage of different dissemina- } \\
\text { tion channels. }\end{array}$ \\
\hline 8 & $\begin{array}{l}\text { 2.4 Multiple communication mediums used for warning dissemina- } \\
\text { tion (e.g. mass media and informal communication). }\end{array}$ & $\begin{array}{l}\text { The ILC and IDC supports the usage of different communication medi- } \\
\text { ums. }\end{array}$ \\
\hline 9 & $\begin{array}{l}\text { 2.6 Consistent warning dissemination and communication systems } \\
\text { used for all hazards. }\end{array}$ & $\begin{array}{l}\text { DEWS and its components have been designed to serve as a multi- } \\
\text { hazard warning system. }\end{array}$ \\
\hline 10 & $\begin{array}{l}2.7 \text { Communication system is two-way and interactive to allow for } \\
\text { verification that warnings have been received. }\end{array}$ & $\begin{array}{l}\text { The IDC reports whether messages have been sent successfully. How- } \\
\text { ever, the reception of emails, text messaging and other warnings is - due } \\
\text { to technical limitations - not guaranteed. }\end{array}$ \\
\hline 11 & $\begin{array}{l}3.1 \text { Warning alerts and messages tailored to the specific needs of } \\
\text { those at risk (e.g. for diverse cultural, social, gender, linguistic and } \\
\text { educational backgrounds). }\end{array}$ & $\begin{array}{l}\text { This is covered by the core concepts of the presented information logis- } \\
\text { tics. }\end{array}$ \\
\hline 12 & $\begin{array}{l}3.2 \text { Warning alerts and messages are geographically-specific to } \\
\text { ensure warnings are targeted to those at risk only. }\end{array}$ & $\begin{array}{l}\text { Administrative units are used to define areas of interest. Only warnings } \\
\text { affecting a certain region are communicated to those at risk. }\end{array}$ \\
\hline 13 & $\begin{array}{l}3.3 \text { Messages incorporate the understanding of the values, concerns } \\
\text { and interests of those who will need to take action (e.g. instructions } \\
\text { for safeguarding livestock and pets). }\end{array}$ & $\begin{array}{l}\text { Message templates can be defined for different user groups with differ- } \\
\text { ent vocabulary. This enables the generation of precise user-tailored } \\
\text { messages providing necessary instructions. }\end{array}$ \\
\hline 14 & $\begin{array}{l}\text { 3.4 Warning alerts clearly recognisable and consistent over time and } \\
\text { include follow-up actions when required. }\end{array}$ & $\begin{array}{l}\text { The system provides functionalities to reference other/older messages. It } \\
\text { is a part of the CAP standard. }\end{array}$ \\
\hline 15 & 3.5 Warnings specific about the nature of the threat and its impacts. & $\begin{array}{l}\text { The ILC supports different message types that have to be threat/hazard- } \\
\text { specific. }\end{array}$ \\
\hline 16 & $\begin{array}{l}\text { 3.6 Mechanisms in place to inform the community when the threat } \\
\text { has ended. }\end{array}$ & The ILC supports the dissemination of All Clear messages. \\
\hline
\end{tabular}




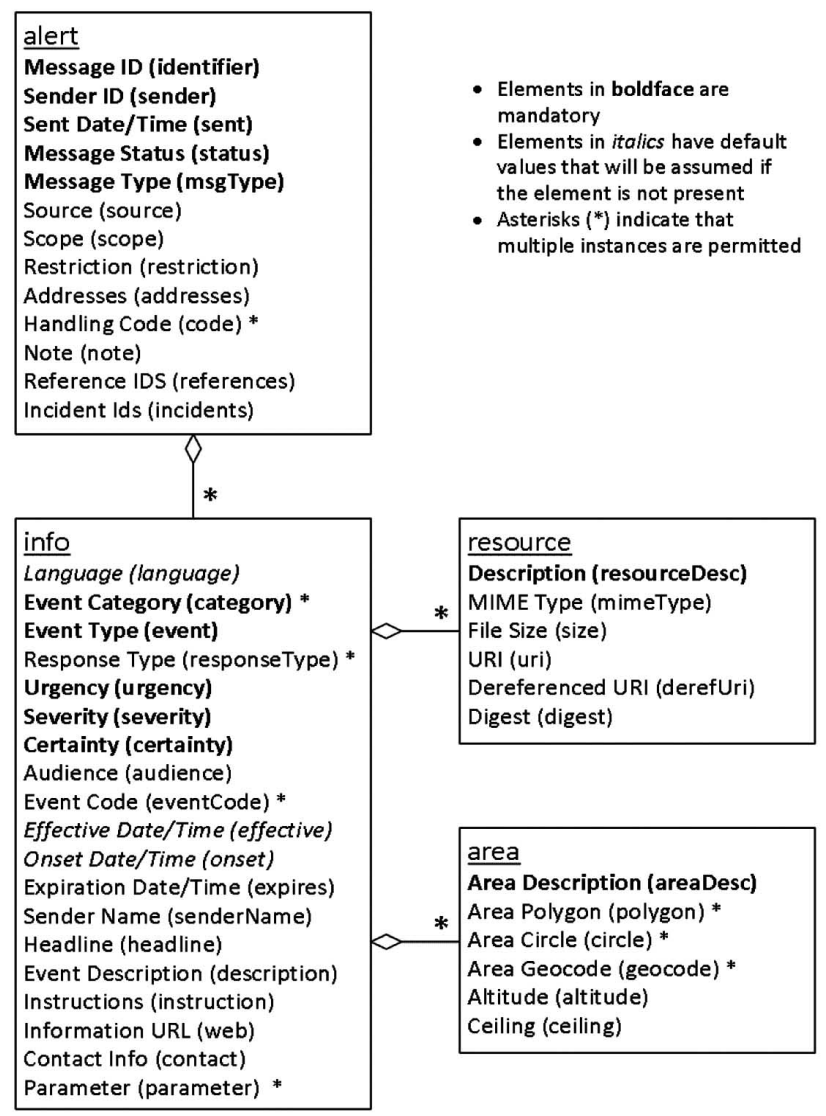

Fig. (2). CAP Document Object Model [16].

instructions about what to do, such as "seek high ground" in a tsunami hazard, whereas rescue services are interested in details of the on-going event. This can take the form of precise estimations about hazard time and severity parameters, e.g., the estimated wave height, the number of wave fronts, etcetera.

- Multilingualism: In most countries, different languages are spoken in parallel without having one language that is mastered by all inhabitants. The support of different languages and different character codes is of fundamental importance for the reasonable usage in a multilingual environment. Otherwise, the notification of and the reception by affected subjects cannot be guaranteed.

\section{THE COMMON ALERTING PROTOCOL (CAP)}

The Common Alerting Protocol (CAP) standard [16] is an XML data format for exchanging information and warnings in emergency situations. CAP has been developed due to the incompatibility of information exchange by different media such as broadcast radio, internet and cellular networks. The standard bridges this communication gap and enables public warning information over a wide variety of data networks and systems. The standard was approved in 2004 by the Organization for the Advancement of Structured Information Standards (OASIS) and has been continuously enhanced since then. The International Telecommunications Unions (ITU) recognised CAP as an emerging global stan- dard for alert and notification systems [17]. CAP is widely used, in particular by American agencies such as the US Department of Homeland Security or by Emergency Services Agencies in Australia [18, 19] but also by EWS such as GITEWS [6], CHORIST [2] and a hazard warning system in Sri Lanka [20]. The concepts behind CAP, its design, history but also the current status and future developments are best described by Botterell [21] and Rohn [22].

A CAP message contains one or more information ("info") elements (see Fig. 2), each compiling all threatspecific attributes for a certain area. Information elements include basic metadata such as date, time or technical message type ("test", "warning", "clear"). Standardised criticality parameters for urgency, severity and certainty enable the classification of emergency situations with pre-defined parameter values. These parameters provide a well-defined manner for addressing question 5 (How intense is the event to be expected?), question 6 (How probable is the event?) and in combination with the "onset" parameter question 4 (When is the anticipated impact?). Hazard-specific attributes can be added as key-value pairs in parameter elements. This structured information, mainly used for automated message exchange and processing, is complemented by human readable information comprising headline, description and instruction. In message processing systems, these three elements are used to generate warning messages in plain text. Spatial references are defined in "area" child elements. Several referencing styles are possible and are discussed in the next section.

To obtain compatibility with other warning systems and with existing automatic message processing systems, the usage of international standards is of the highest priority. Comparable alternatives do not exist, and the suitability of CAP has been approved in several warning and notifications systems.

\section{SPATIAL REFERENCE IN EARLY WARNING MES- SAGES}

The spatial reference of warning messages is of utmost importance for the message recipient. Hazard details such as severity or estimated onset are valueless if the spatial reference is either missing or cannot be interpreted correctly. Human recipients must be able to assess if they are endangered or not and to build a situational picture to define their individual course of action.

CAP offers three alternatives for spatial referencing, each with specific pros and cons. References can be established by either defining perimeters (centre and radius), defining arbitrary polygons (lists of vertices) or by addressing (political) areas/regions with standardised geocodes. Describing the affected area of a hazard with a single circle will reflect the reality only in rare cases and is only suited for hazards that have a well-defined centre and whose criticality declines with distance from the centre, for example earthquakes. The specification of a polygon of arbitrary complexity enables the precise definition of the affected area, such as the surface of an oil spill or the lava flow from an erupting volcano, but also areas threatened by a tsunami. However, human perception of this spatial reference is reduced with increasing com- 


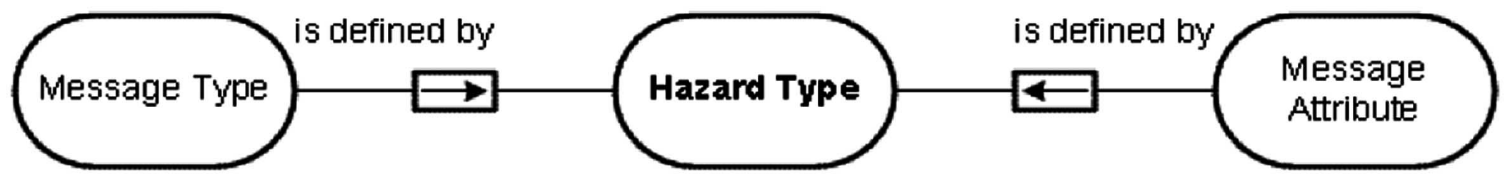

Fig. (3). The Hazard Type entity and its relation to Message Type and Message Attributes

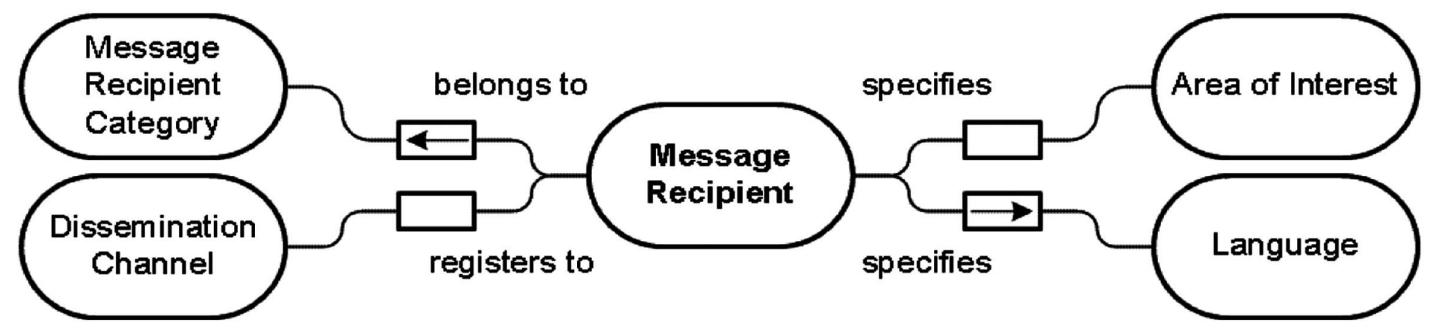

Fig. (4). The Message Recipient entity and its relations.

plexity of the polygon. Humans are not normally aware of their current position in terms of coordinate values, and it is even less possible to identify the shape of the polygon and which parts of the real world are inside or outside. Thus, processing the polygons must be done with tools and geographic information systems (GIS) that display the polygons' shapes and positions in a human-readable manner. In contrast, addressing areas/regions with geocodes for territorial subdivisions, such as federal states and counties, provides a spatial reference that is directly usable for all message recipients. The mapping of spatial references to administrative units in EWS is examined in detail in [31]. Hierarchical administrative territories are structured according to historic cultural and ethnic landscapes or logistics districts that are commonly known and widely used. Thus, civilians directly know if they are located in the affected area or not. Because structures and hierarchies of governmental agencies are often aligned with political subdivisions, this usage also fits with the business aspects of these services. Limitations based on the impreciseness of administrative units as affected areas can be softened with the combined usage of geocodes and precise polygons. Consequently, the parallel usage of geocodes (for human readable message parts) and precise polygon definitions (for automated processing) combines the benefits of both approaches. Moreover, this enables and supports the usage of broadcasting dissemination channels for which the vertices have to be converted into the Universal Geographical Area Definition (GAD) [23]. CAP does not request a specific geocode standard to be used; instead, areas are addressed by one or more key-value pairs in which the key defines the geocode standard and the value contains the geocode. Different geocodes based on different standards, such as ISO-3166, or Secondary Administrative Level Boundaries data sets (SALB) can be used alone or in combination with each other. However, the generated warning messages must also include the real name of the administrative unit since code recognition might not be expected from all message recipients.

\section{CONCEPTS OF GENERIC INFORMATION LOGIS- TICS}

The field of information logistics aims at developing concepts, technologies and applications for need-oriented information supply. Information-on-demand services are a typical application area for information logistics, as they have to fulfil user needs with respect to content, location, time and quality [3]. Because most EWS focus on the upstream part and neglect the downstream part, the realisation of information logistics should provide a flexible design and decoupled implementation, allowing easy adoption and integration.

Analysis of the requirements reveals that the central task of information logistics is the generation of user-tailored warning messages. In a typical hazard scenario, different phases are encompassed; initial physical events are followed by observations, forecasts and decision-making. These phases and the alarm level must be reflected by different message types. For example, during a tsunami threat following an initial earthquake, "Heads-Up" messages are disseminated, which inform of the possibility of a triggered tsunami. These are followed by "Tsunami Warning" messages based on observed wave propagation or "All Clear" messages if no tsunami is generated. Other hazards might require other message types. Message Types (see Fig. 3, left) are hazardspecific and are defined by the Hazard Type (see Fig. 3, middle). The Message Type is a statement such as "All Clear Message". The message content describes the situation with on-going and up-to-date information. It is a mixture of hazard type independent values such as onset time or affected area and hazard type specific attributes such as tsunami wave height or radioactive contamination value. The Hazard Type defines both the available Message Types and Message Attributes (see Fig. 3, right), which are added as key value pairs into the CAP message.

The Message Recipient (see Fig. 4, middle) is the subject that receives the disseminated messages from the EWS. This can be an individual (for example, a civilian or the governor of a province) or an institution, such as rescue services or a local warning authority (for example, a beach watchtowers). This grouping is realised with Message Recipient Categories (see Fig. 4, top left), which handle group-wide settings such as filtering (see next paragraph). The Message Recipient defines the preferred Language in which he wants to receive the messages, the Area of Interest of which he wants to be informed and the Dissemination Channels he makes use of, as indicated below: 


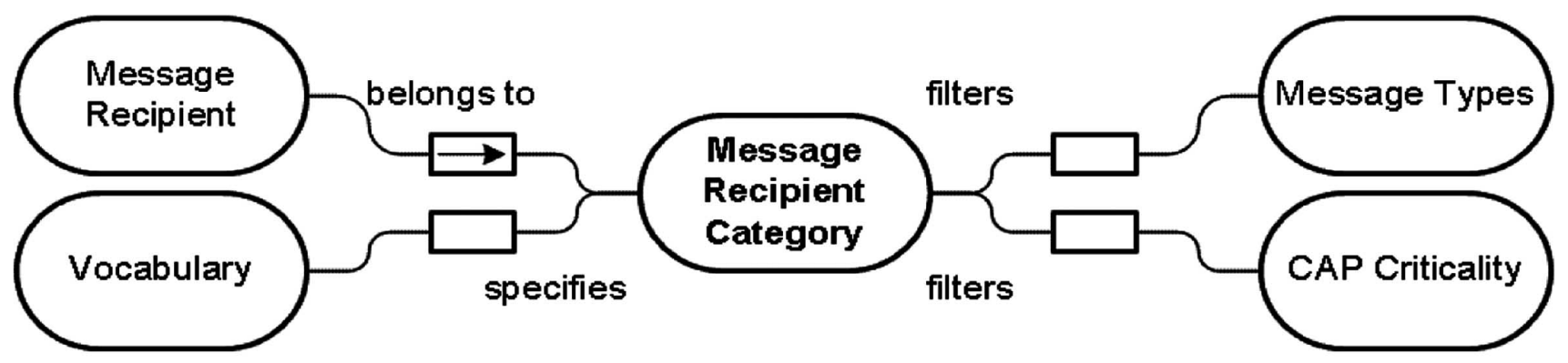

Fig. (5). The Message Recipient Category entity and its relation to Filter Types.

- Language (see Fig. 4, bottom right) preference is specified by ISO 639-1 codes [24], for example, "en" for English or "si" for Sinhala. As mentioned earlier, the request for multilingualism is accompanied by the request for the support of different character sets. This is implemented by supporting Unicode Transformation Format (UTF).

- Area of Interest (see Fig. 4, top right) is defined by one or more geocodes that address administrative areas and matching affected areas identified in a threat.

- Dissemination Channels (see Fig. 4, bottom left) are specified by Message Recipients, typically those channels that subjects want to use to transmit information and that are most efficient in an emergency situation. Civilians, for example, will register for text message or email alerts but not via their workplace fax number.

Message Recipients belong to a certain Message Recipient Category (see Fig. 4, top left and Fig. 5, middle) such as rescue services or the general public. To ensure that all message recipients belonging to the same group are informed under the same conditions, all message filters - except for the Area of Interest - are linked to the Message Recipient Category and not to the Message Recipient.

- One filter specifies the Message Types (see Fig. 5, top right) that are of interest for the category.

- There are three filter matches on the three aforementioned CAP Criticality (see Fig. 5, bottom right) values (urgency, severity and certainty).Thresholds are defined for each Message Recipient Category and must be reached or exceeded in order to be transmitted.

- In addition to the contributing filter mechanisms, a Message Recipient Category specifies the Vocabulary (see Fig. 5, bottom left) requested for all of its messages. This allows for different levels of knowledge and terminology that account for different Message Recipients.

All filters (area of interest, message type, CAP severity, CAP certainty and CAP urgency) defined by the Message Recipient and its related Message Recipient Category are applied one after another during the message generation process, and all have to be matched to trigger message dissemination. Message generation is only performed if all the filters match.
The CAP elements Title, Description and Instructions constitute the human readable part of the message. These elements are not generated manually or on-the-fly during message generation. Instead, predefined Message Templates are used. A template is addressed and selected by message type, vocabulary, language and dissemination channels (see formula 1 ).

$f$ (Message Type, Vocabulary, Language, Dissemination Channel) $\rightarrow$ Message Template (1)

The translation into different languages could alternatively be realised with an on-the-fly translation service. Although such translation services are the subject of research projects, the reliability and accuracy for EWS is not sufficient. The CHORIST project investigated automatic template translation, though the results thus far are not convincing [2]. The fourth key (Dissemination Channel) has been added to incorporate dissemination channel specific limitations such as character encoding (for example, insufficient UTF support for the fax or text message receiver) or text length limitations (for example, 160 characters maximum for a text message).

To enrich message templates with details of the on-going hazard, placeholders are used that enable on-the-fly replacement during message generation:

- Criticality placeholders: severity, certainty and urgency. These are based on the corresponding CAP elements and use the same predefined values. Their situation-specific values are based on the characteristics of the on-going event.

- Spatial reference: corresponds with geocodes as used in the area elements of the CAP message.

- Time: corresponds with the onset parameter defined in the CAP message.

- Hazard-specific placeholders: these are based on the hazard-specific message attributes that are also included as key value pairs in the CAP additional parameters section.

All generated messages are converted into channelspecific encodings and protocols before being disseminated to the message recipients. Based on user preferences and the dissemination channel capabilities, either the human readable parts or the whole CAP is communicated. This feature is of interest for recipients using automated (CAP-) processing systems. Channel characteristics such as the character limitation in text messages may overrule this preference. 


\begin{tabular}{|c|c|c|c|c|}
\hline \multirow{5}{*}{$\begin{array}{l}\text { Message Recipient } \\
\text { Category } \\
\text { e.g., Rescue Service, } \\
\text { General Public, ... }\end{array}$} & \multirow{5}{*}{$\begin{array}{l}\text { specifies its } \\
\text { vocabulary } \\
\text { defines filter } \\
\text { for message types } \\
\text { defines filter } \\
\end{array}$} & \multirow{2}{*}{$\begin{array}{c}\text { Vocabulary } \\
\text { e.g., Basic, Medium, Detailed }\end{array}$} & \multirow{4}{*}{$\begin{array}{r}\text { defines } \\
\text { message types }\end{array}$} & \multirow{5}{*}{$\begin{array}{l}\text { Hazard } \\
\text { Type } \\
\text { e.g., } \\
\text { tsunami, } \\
\text { volcanic, } \\
\text { land slide }\end{array}$} \\
\hline & & & & \\
\hline & & $\begin{array}{c}\text { Message Type } \\
\end{array}$ & & \\
\hline & & e.g., Heads Up, Tsunami Warning, All Clear & & \\
\hline & & $\perp$ & & \\
\hline belc & threstioic & Urgency, Severity, Certainty & & \\
\hline & & $i$ & templa & \\
\hline & specifies its & Area of Interest & Hazar & pecific \\
\hline $\begin{array}{l}\text { Message Recipient } \\
\text { e.g., Province Hospital, }\end{array}$ & area of interest & e.g., Phuket: ISO_3166-2 = TH-83 & Message & tributes \\
\hline $\begin{array}{l}\text { Watchtower at beach, } \\
\text { Tourist }\end{array}$ & & Language & $\begin{array}{l}\text { Key value } \\
\text { estimated ti }\end{array}$ & $\begin{array}{l}\text { irs, e.g., } \\
\text { of arrival }\end{array}$ \\
\hline & preferred language & e.g., English: ISO_639-1 = en & (eta), esti & ted wave \\
\hline is $\mathrm{re}$ & & T & height ( & Cw \\
\hline & & ...... template & & \\
\hline Dissemination & & $\boldsymbol{v}$ & & \\
\hline $\begin{array}{l}\text { Channel } \\
\text { e.g., SMS, FAX, Email, } \\
\text { TV Broadcasting }\end{array}$ & $\frac{\text { channel specific }}{\text { conversion }}$ & $\begin{array}{l}\text { Message Templat } \\
\text { e.g., Tsunami Warning, Vocabulary: Med } \\
\text { Tsunami Warning! Your location (\$location) is threatened } \\
\text { ETA: \$eta. Wave Height: Sewh. Seek immediately shelter! }\end{array}$ & $\begin{array}{l}\text { Language } \\
\text { Tsunami. Sev }\end{array}$ & sh \\
\hline
\end{tabular}

Fig. (6). Overall Information Logistic Concept for early warning systems.

Fig. (6) summarises the overall information logistics concept.

\section{REALISATION AND IMPLEMENTATION}

The Distant Early Warning System (DEWS) project was funded in the $6^{\text {th }} \mathrm{FP}$ of the EC. A well-balanced consortium of public and private organisations from several EU member states, as well as partners from around the Indian Ocean, have worked together closely in order to design and implement an open, standard-based EWS realising both reliable hazard detection and effective warning dissemination [25]. Within the project, a reference architecture for EWS has been developed; its hazard-independent and generic information logistics are presented here.

\section{SYSTEM ARCHITECTURE}

The simplified architectural blueprint (see Fig. 7) provides an overview of DEWS following the principles of SOA (Service Oriented Architecture). A sensor network, map services and simulation systems - together compiling the upstream information flow - are connected to the Command and Control User Interface (CCUI) using standardised OGC services. The downstream, including the message dissemination from CCUI via the Information Logistics Component (ILC) and Information Dissemination Component (IDC) to the message recipients, has been realised with web service technology us- ing SOAP (Simple Object Access Protocol).

\section{WARNING MESSAGE GENERATION}

The message generation process is divided into three phases and components (see Fig. 8):

1. The CCUI releases an initial threat-specific CAP warning message. For each affected area, one CAP information element is generated that contains all area-specific attributes, such as the estimated time of arrival (ETA) and estimated wave height (EWH). These CAP messages are independent from specific user settings and do not provide the headline, description and instruction elements.

2. The ILC generates one customised CAP message for each user and the respective dissemination channel, as described in Fig. (9):

a). All affected message consumers are identified according to their areas of interest.

b). For each user and for each of its effective areas, all filters are applied.

c). Templates for all relevant dissemination channels are generated, respecting the message consumer's preferred language and vocabulary defined by the category he or she belongs to.

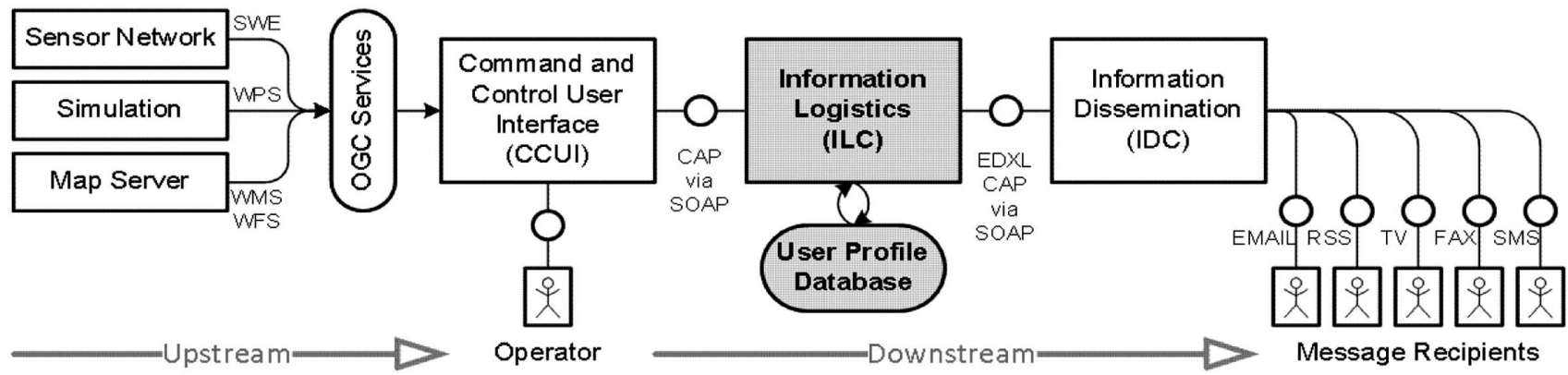

Fig. (7). Information Logistics Component (ILC) in the DEWS reference architecture. 


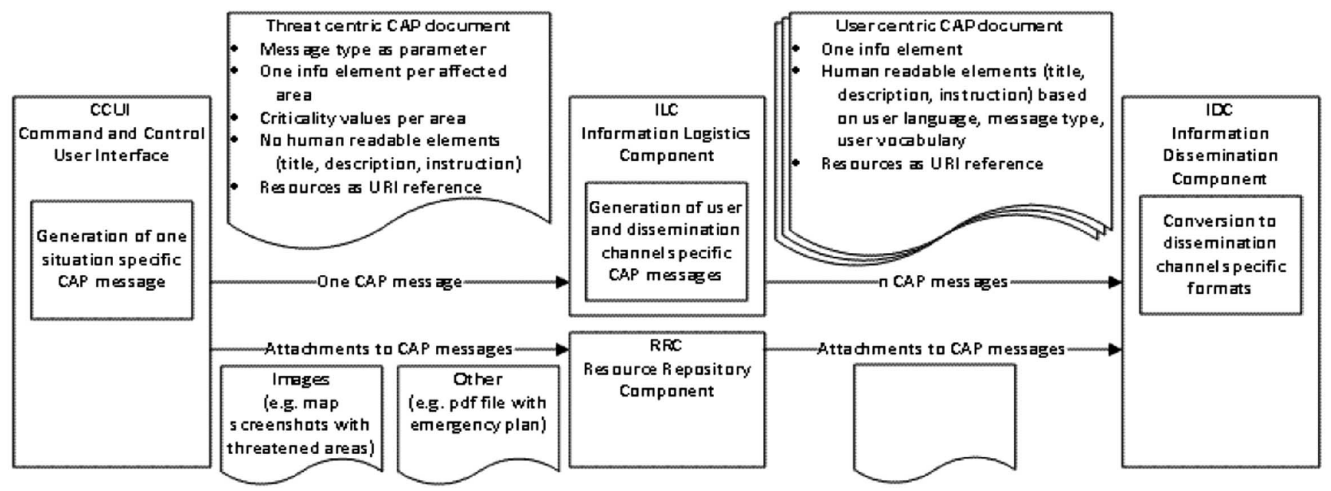

Fig. (8). Flowchart of the CAP message generation process consisting of threat centric messages generated by CCUI and user centric messages generated by ILC.

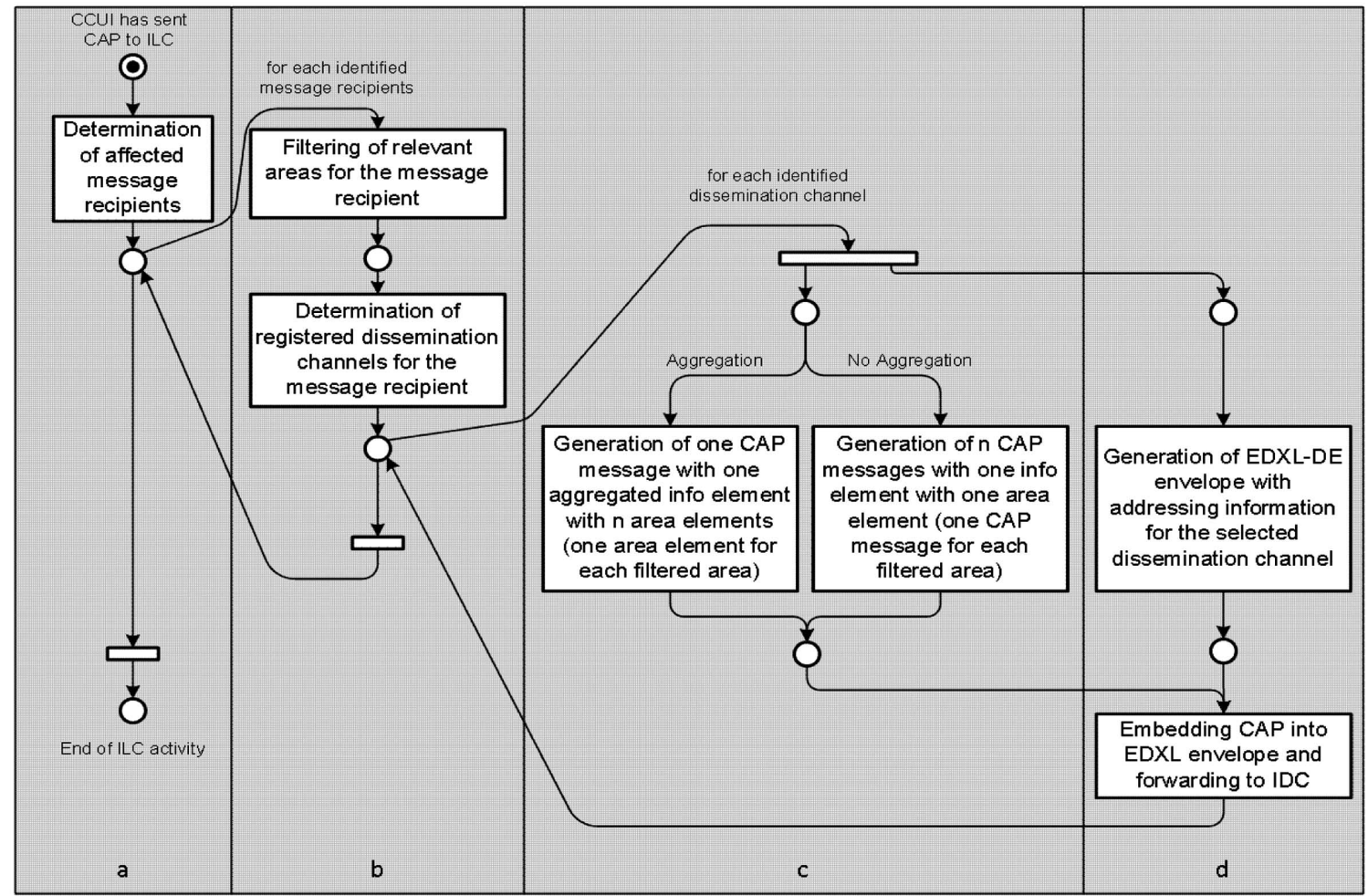

Fig. (9). Generation of user-specific warning messages in Information Logistics (Notation: Petri Net).

Place-holders are replaced with area-specific values.

d). Generated CAP messages are embedded into an EDXL-DE (Emergency Data Exchange Language Distribution Element) envelope [26] equipped with addressing data.

3. The IDC converts the EDXL-DE/CAP messages into channel-specific formats and finally disseminates them to the message recipients.

The implemented information logistics comes with the following advanced characteristics to improve the message generation and reception process:

- Aggregation of warning messages: A message consumer registered to more than one area of interest would receive one warning message for each area.
This could lead to spam-like message flooding, frustrating the message recipient. The aggregation functionality aggregates all warning messages addressed to the same message consumer. Inside the message templates, the event and area specific attributes like geocode or time of arrival are concatenated.

- Sub-region-Aggregation: Political subdivisions are hierarchically ordered. A message recipient registered for a certain region would be informed several times if more than one area of lower order is affected. This could also lead to unintended message flooding. Instead, one warning message will be generated with hazard specific attributes based on worst-case combinations of all sub-regions, for example, the highest EWH of all affected lower level regions is taken as the super-region. 


\begin{tabular}{|c|c|c|}
\hline & Template in database & Generated CAP elements \\
\hline Headline & Alerta de tsunami & $<$ headline $>$ Alerta de tsunami $</$ headline $>$ \\
\hline Description & $\begin{array}{l}\text { Inminente llegada de un tsunami. } \\
\text { Ubicación <Dews:AffectedLocation } \\
\text { type="HASC"/>: } \\
\text { ETA=<Dews:MinTimeofArrival/>, } \\
\text { max SSH=<Dews: MaxSeaSurfaceHeight/>, } \\
\text { Certeza=<Dews: Certainty/>, } \\
\text { Gravedad=<Dews: Severity/>, } \\
\text { Urgencia=<Dews:Urgency/>. }\end{array}$ & $\begin{array}{l}\text { <description>Inminente llegada de un tsunami. } \\
\text { Ubicación TH.PG.TT. Khok Kloi: ETA=2010-06- } \\
\text { 03T09: } 45: 22+0000, \text { max SSH=5.43, Certeza= } \\
\text { probable, Gravedad = extrema, Urgencia }= \\
\text { prevista.</description> }\end{array}$ \\
\hline Instruction & $\begin{array}{l}\text { Proclame fase de alerta 2. Informe } \\
\text { al público y autoridades locales. } \\
\text { Prepárese para la llegada del } \\
\text { tsunami. observe continuamente la } \\
\text { evolución de la situación. }\end{array}$ & $\begin{array}{l}<\text { instruction>Proclame fase de alerta } 2 \text {. Informe } \\
\text { al público y autoridades locales. Prepárese para la } \\
\text { llegada del tsunami. Observe continuamente la } \\
\text { evolución de la situación.</instruction> }\end{array}$ \\
\hline
\end{tabular}

Fig. (10). Comparison of message template as stored in the ILC database (left column) with the generated CAP elements (right column) enriched with situation - specific attributes such as location, time of arrival and wave height.

- Translation of CAP criticality values: The CAP standard includes pre-defined values for each criticality element, for example, "Extreme", "Severe", "Moderate", "Minor" and "Unknown" for severity. Using these terms in the placeholders generates languagebreaks in non-English messages. This could lead to confusion and misinterpretation. Therefore, translations of these values are used within placeholders whereas the CAP elements itself are not changed since this would violate the standard.

- CAP-Include: Message recipients have the option to receive the full CAP document in addition to the generated template-based message. This feature is of interest for recipients using automated (CAP) processing systems.

A comparison of a prepared template and the corresponding generated CAP elements is given in Fig. (10). It outlines how the initial template with its placeholders is converted into a threat-specific warning message.

\section{GUI DESIGN}

The graphical user interface facing the operator plays a central role in the software suite of EWS. It must fulfil strict requirements regarding reliability, safety and usability. It must also provide a clear and intuitive interface for operators and must not be overburdened with functionality or distracting elements. In the reference architecture developed within the DEWS project, the CCUI was designed, like all other components, to serve as a reusable component in multi hazard scenarios. Therefore a modular design was chosen. The tree-like dependency structure enables the easy integration of new functionality without modifying plug-ins, providing the core functionality such as CAP generation. On the other hand, these core modules must be flexible enough to comprehensively provide basic message generation functionality via forms and other GUI components but must also accept generated CAP fragments from other plug-ins serving as bridge to the ILC. Such modular extendibility has been realized by method hook-ups, static classes following the Singleton-pattern [27] and by providing Extension Points that are a central concept of the Eclipse RCP architecture [28]. Fig. (11) depicts the layered architecture of the CCUI.
Library plug-ins provide basic functionality for all other GUI plug-ins such as a communication stub towards the ILC and a representation of the CAP schema as object types. The core ILC plugin offers a complete GUI perspective (see Fig. 3) dedicated to the generation of CAP messages and related functionality. Plug-ins sitting atop the ILC plug-in provide hazard specific functionalities. The topmost level of all plug-ins contributes project or deployment specific functionalities that are bound to a certain setup and are not sufficiently generic to be located in other plug-ins.

\section{CORE INFORMATION LOGISTICS FUNCTIONAL- ITY IN CCUI}

The ILC plug-in of the CCUI has been designed to provide an all-encompassing set of functionalities for the generation of CAP warning messages. Fig. (12) displays the Message Generation Perspective, which does not provide any hazard or deployment specific extensions and can therefore be re-used in a multitude of application scenarios.

The various interface elements provide a high degree of freedom, not forcing the operator to follow a strict step-bystep procedure to disseminate a warning message. Instead, sufficient flexibility enables different workflows depending on the ongoing situation.

For the classification of an affected area, the proper tool has to be selected from the toolbar (A1). Each vertex is added by a single click to the map (A2). With a double click, the geometry is finalized and automatically analyzed. If it is a valid and not self-intersecting polygon, the new area is added to the Affected Areas view part (A3). In the background, a Web Processing Service (WPS) is triggered to resolve all intersected administrative territories. The area information is updated automatically and results of this process such as geocodes of administrative units ("TH.PG.TM" for the region Thai Muang) are displayed in the Detail View (A4).

To enrich warning messages with a situation picture, screenshots of the map can be taken. This is done by a single click on the corresponding tool (B1). The new screenshot appears in the Snapshots view part (B2). Further options like renaming or cropping the image are available from the context menu that opens by right-clicking on the screenshot (not shown in Fig. 3). 


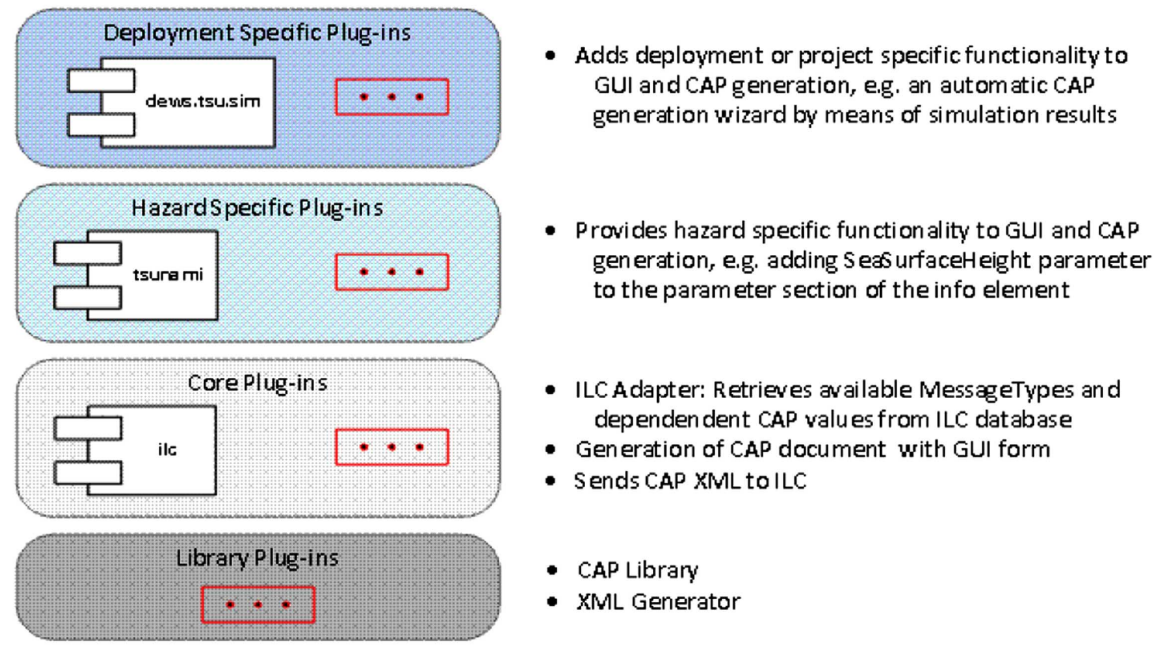

Fig. (11). CCUI plug-in dependency stack responsible for CAP message generation.

The Message Generation part provides a form for message creation. As noted above, the CCUI will generate messages in the CAP format and send it to the ILC. The form's design follows segments of the CAP standard without confronting the operator with technical details. The most important decision that is made by the operator is the selection of the proper message type. As defined in Fig. (3), message types depend on the hazard type. Selectable message types are provided by the ILC database, which has to be set up for each deployment. This database provides all deploymentspecific settings, message types and templates and is presented in [32]. After message type selection (C1), the value ranges of all associated fields (see Table 1) are updated automatically. In the example above, the choices for Category (C2) have been limited to "Safety" and "Geo" and the Status (C3) has been set to "Actual" without being modifiable since it is the only value that is allowed for this field according to the definition of the message type "Tsunami Warning" in the ILC database. The criticality values urgency, severity and certainty $(\mathrm{C} 4)$ have to be set by the operator. By default they are set to the less critical value allowed for the selected message type. The operator can add additional parameters as key value pairs to the message within the Additional Parameters section (C5). A modal dialogue opens when pressing the plus button. Several predefined attributes are selectable from a drop-down list or new parameters can be added. An internal extension point allows contribution of pre-defined attributes by other plugins. This extension point also includes a mechanism to add validator functionality limiting the allowed values, for example the tsunami plug-in adds the parameter Sea Surface Height, together with a validator checking that only decimal numbers are set. Affected areas can be added to the Affected Areas section (C6) in multiple ways. They can be copied via drag and drop from the Affected Areas view part (A3), with a context menu action provided also by this view part or from a selection list that opens by pressing the plus button to the Affected Areas section. Although the CAP standard foresees different criticality values for each region (they are defined per information element), the form has been designed to be simple. It avoids extra dialogues and sub-forms where possible. Therefore all information elements (one for each affected area) generated by this form will have the same criticality values and parameters. If different values and parameters are set for the selected areas, then multiple messages, one for each area, must be generated. For this purpose, a context menu action in the Disseminated Messages view part (D1) provides the functionality to (re-)use a disseminated message as a boilerplate. This option significantly limits the effort needed to create messages for different areas with only a few diverging attributes. Screenshots are attached to the message by the same options as affected areas: either via drag and drop, or via context menu action or by using the selection dialogue that opens after pressing the corresponding "plus" button in the Snapshots section (C7). Analogous referencing of older messages has been realized; they can be added to the Message References section (C8). However, the ILC will automatically create message references for messages previously sent within the context of the same incident.

The message is finally released by activating the "Start Message Dissemination" button (C9). An information field informs (C10) the operator during form handling of any inconsistencies, errors or any other suspicious settings (for example, no affected area selected). Released messages are listed in the Disseminated Message view part (D1) which is automatically updated if message processing has been finished by the ILC. Details of the IDC message processing, for example dissemination statuses, are listed within the Dissemination perspective (not shown in Fig. 11), which provides detailed information about each disseminated user message in separate views.

\section{TSUNAMI EARLY WARNING EXTENSION}

As stated in the introduction, the overabundance of data might limit human capabilities to disseminate timely warning messages. In the event of a tsunami the ocean-wide coastline might be affected, resulting in hundreds of affected administrative units. To combat this information overflow, tsunami-specific modules have been developed, which automatically generate warning messages for each affected unit. In the scope of the DEWS, project simulations provided by the Alfred Wegener Institute have been integrated which 


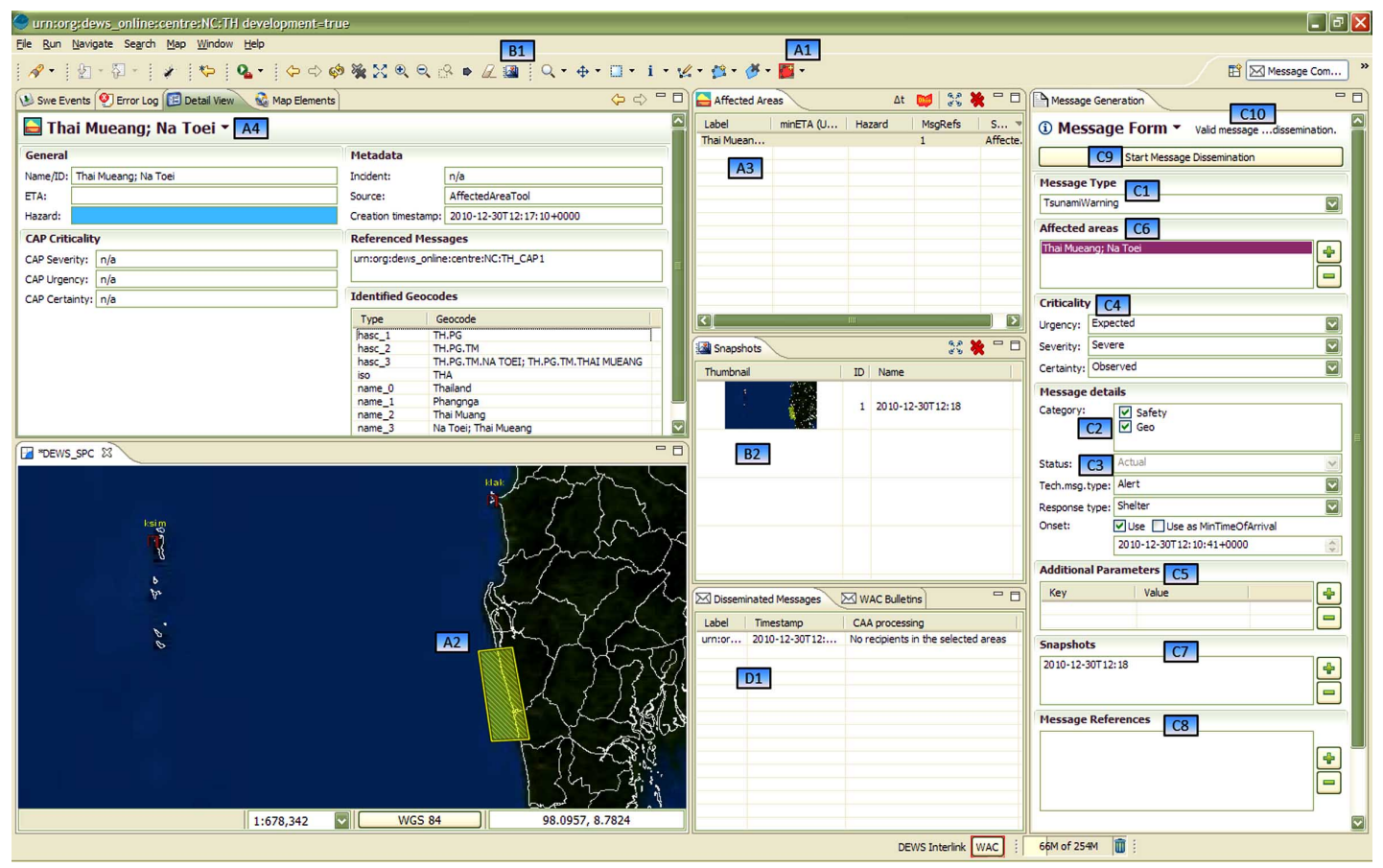

Fig. (12). CCUI Message Composition Perspective.
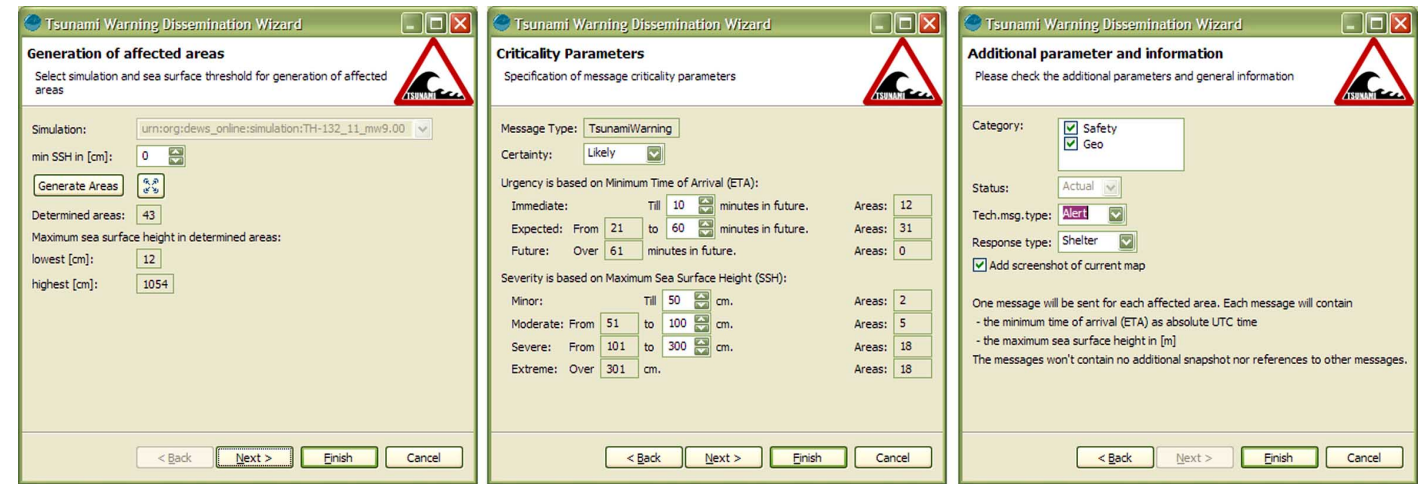

Fig. (13). Three step Tsunami Warning Dissemination Wizard supporting the operator with the generation of simulation-based CAP messages. 13a: Identification of affected areas by background WPS, 13b: Determination of urgency and severity by means of simulation results, 13c: Further CAP settings and option to add a screenshot.

provides forecasts for run-up heights and arrival times on the basis of real-time sensor measurements [29] according to the IOC requirements for Regional Tsunami Watch Provider (RTWP) [30]. The simulation system provides different kinds of products such as isochrones visualizing wave propagation or tide gauge level predictions for comparison with real-time sensor measurements. For the identification of threatened areas, simulation products have been used that include ETA and EWH for pre-defined points along the coastline. Apparently it will be impossible for operator(s) of a CIMS to analyze and assess the predictions for hundreds or even thousands of coastal points. A GUI component named "Tsunami Warning Dissemination Wizard" (see Fig. 4) realizes the automatic processing and CAP message generation. However, the responsibility remains in the hands of the operator who ensures all messages are released by finishing the wizard.

The first page of the Wizard (see Fig. 13a) provides functionality for the identification and classification of adminis- trative units. This triggers a WPS that maps forecasts to territorial units provided by Spatial Data Infrastructure (SDI). See [31] for details. The second page (see Fig. 13b) serves to assess the criticality parameters. For each affected area and thus for each corresponding CAP information element, the Urgency classification is based on the predicted ETA. Predefined classification thresholds can be adjusted by the operator, resulting in different groupings. While the wizard is open, a background task updates the form every second and re-classifies all areas based on the present time. The Severity classification is based on the second simulation parameter, the predicted sea surface height (SSH, identical to EWH). On the third page of the wizard, remaining CAP attributes such as Category or Status might be specified. Most of them are already limited by the automatically chosen message type. Also, the automatic addition of a screenshot can be selected on this page. Finally, by releasing all messages, the wizard generates one CAP message for each affected area with its specific criticality values and ETA and SSH parameters. 
Table 3. Validation Against Open Questions for Constructing a Warning Message

\begin{tabular}{|c|c|}
\hline Open question & Validation \\
\hline 1. Who is issuing the warning? & $\begin{array}{l}\text { The message sender is set in the CAP sender ID. It can also be part of } \\
\text { the message template that will be used for the human readable warning } \\
\text { message. }\end{array}$ \\
\hline 2. What type of event is threatening? & $\begin{array}{l}\text { The type of event is encoded in the message type (such as "tsunami } \\
\text { alert" or "earthquake information"). It defines the selected message } \\
\text { template and can also part of the message template. }\end{array}$ \\
\hline 3. Who is being threatened? & $\begin{array}{l}\text { The selection of message recipients follows strict rules based on de- } \\
\text { fined areas of interest and additional message filters. }\end{array}$ \\
\hline 4. When is the anticipated impact to occur at recipient's location? & $\begin{array}{l}\text { For each affected area, the CAP onset time is set either manually or } \\
\text { automatically. Within message templates, placeholders can be used to } \\
\text { activate the insertion of estimated time of arrival in message body. }\end{array}$ \\
\hline 5. How intense is the event expected to be at the warning recipient's location? & $\begin{array}{l}\text { CAP severity parameter is used for this threat classification. Within } \\
\text { message templates, placeholders can be used to activate the insertion of } \\
\text { the estimated severity in message body. }\end{array}$ \\
\hline 6. How probable is it that the event will strike the warning recipient's location? & $\begin{array}{l}\text { The CAP certainty parameter is used for this threat classification. } \\
\text { Within message templates, placeholders can be used to activate the } \\
\text { insertion of estimated certainty in the message body. }\end{array}$ \\
\hline 7. What specific protective actions should be taken? & $\begin{array}{l}\text { Clear instructions can be defined for each user group separately by } \\
\text { defining special message templates. }\end{array}$ \\
\hline 8. Are there high-risk groups that require special actions? & $\begin{array}{l}\text { For high-risk groups, special message templates can be defined by } \\
\text { special instructions or providing a special vocabulary. }\end{array}$ \\
\hline
\end{tabular}

Upon releasing all CAP messages, the wizard is closed and details of the classified areas are shown in the Message Composition Perspective (see Fig. 14). Affected areas are coloured by the predicted threat level in accordance with the colour scheme recommended by the US Department of Homeland Security [34]. They are listed with the predicted tsunami ETA and SSH in the Affected Areas view part. Since each area represents one administrative territory, the Geocode section of the Detail View provides one entry for each level of the territorial hierarchy.

\section{VALIDATION \\ UN/ISDR Checklist}

Validation of the developed concepts is performed against the UN/ISDR checklist for early warning systems. All applicable checklist items (see Table 1) are taken into account. In any case, the overall system architecture developed within the DEWS project will be validated against the whole checklist.

Many items on the checklist address issues that deal with the concrete deployment of an early warning system and cannot be answered on the basis of system components only. However, the checklist indicates that all applicable items are achieved by the presented concepts and it indicates that components developed within the DEWS project could serve as reference architecture incorporating ISDR requirements for EWS. But it must be clearly stated that the way these components are used in a concrete deployment determines whether a successful early warning system will be achieved or not. For example, the definition of meaningful and comprehensive message templates is a critical task that must be performed for each installation based on national, cultural but also threat-specific backgrounds, preferably by a communication professional.

\section{WARNING MESSAGE REQUIREMENTS}

A second validation is performed by matching the capabilities against the open questions for warning messages raised by Lindel and Perry [11]:

As shown in Table $\mathbf{3}$, all open questions can be answered successfully. The presented concepts offer technologies for the definition of precise message templates for arbitrary target groups with their individual requirements, different levels of understanding, distinct perceptions and varying personal abilities.

\section{FEEDBACK}

The concepts of information logistics presented here have been demonstrated in project-internal and public DEWS live demonstrations. In particular, the support of arbitrary languages in combination with UTF gained much attention (see Fig. 15). This has been a major requirement of project partners from Thailand, Sri Lanka and Indonesia. Furthermore, the generation of user-tailored warning messages using adequate terminology, in combination with the selected level of detail based on template placeholders has been assessed positively.

A professional review and assessment of the overall system was carried out with domain experts at the Meteorology, Climatology and Geophysics Agency (BMKG) in Jakarta, Indonesia and at the Kandilli Observatory and Earthquake Research Institute (KOERI) in Istanbul, Turkey. Moreover, 


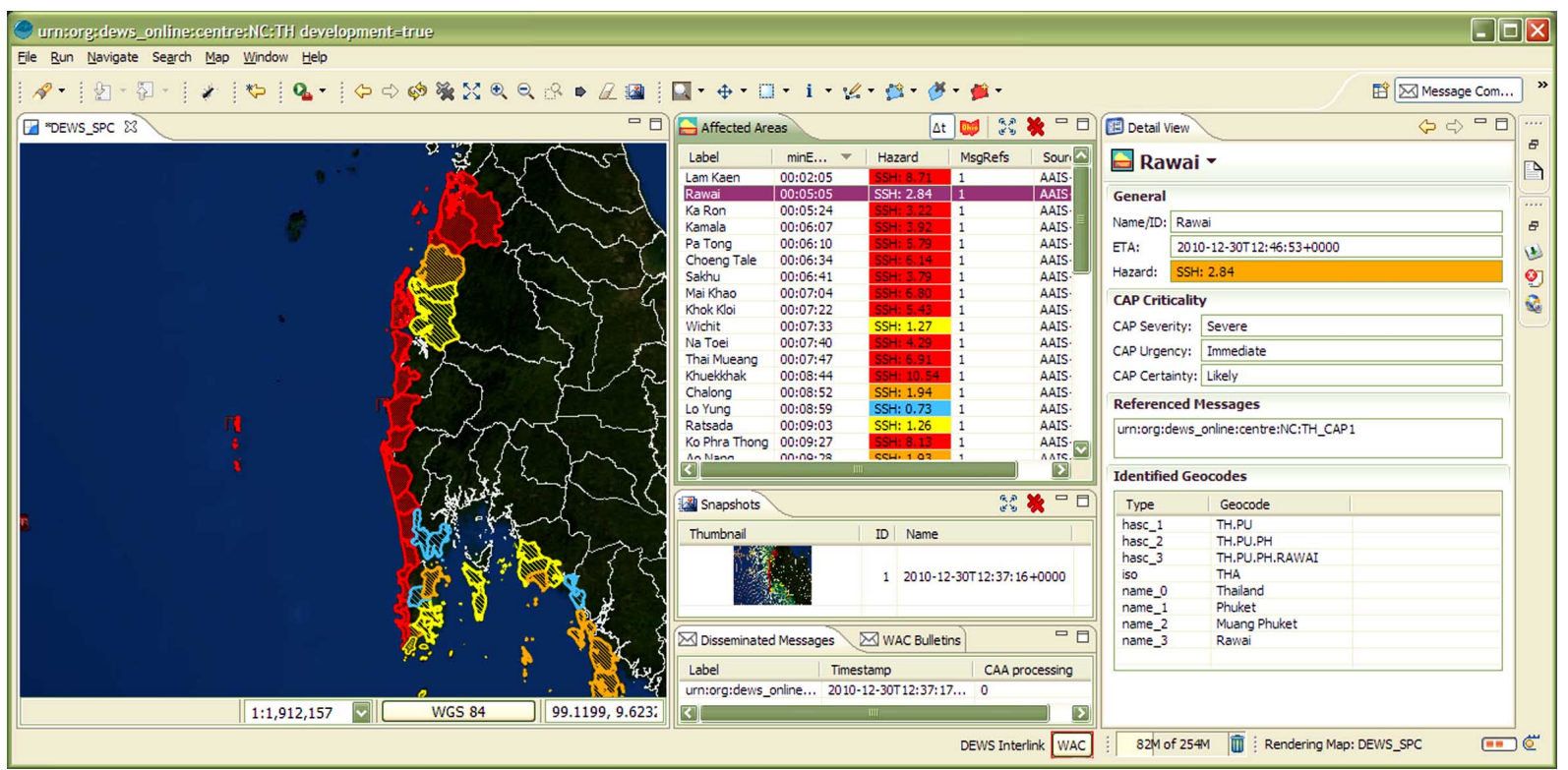

Fig. (14). CCUI Message Composition Perspective with automatically generated affected areas.

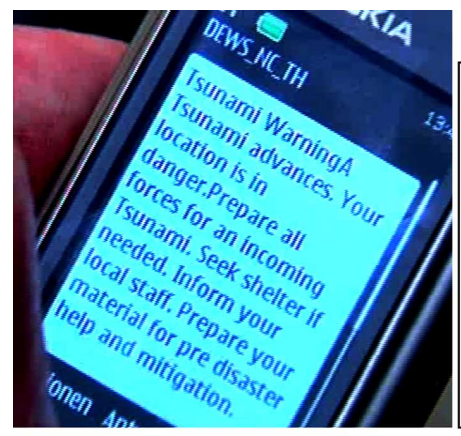
சுனாமி எச்சரிக்கை!
¿ DEWS Consortium
சுனாமி வந்துகொண்டிருக்கிறது பிரதேசம்TH.PG.TM.Thai Mueang: எதிர்பார்க்கப்படும் வருகை நேரம் =2009-09-28T09:56:07+0000, ஆகக்கூடிய அலை உயரம் $=6.9$
சுனாமி எச்சரிக்கை இருப்பதனால் பாதுகாப்பான இடங்களிற்கு நகரவும் உள்ளூர் அலுவலர்க்கு அறிவிக்கவும். வெளியேற்று நடவடிக்கைகளை பின்பற்றவும்.

Fig. (15). Examples of generated tsunami warnings during live DEWS demonstrations. Left: A text message in English informing local rescue services using basic instructions. Right: email in Tamil including geocode, estimated time of arrival and estimated maximum wave height for the affected territory.

the installation at BMKG was used for a system evaluation in parallel with the Indian Ocean Tsunami Warning and Communication Exercise 2011. Review, validation strategy and feedback are thoroughly described in [33] which covers not only the ILC but the overall DEWS.

The feedback was consistently positive. Primarily, the seamless component integration into one early warning system in combination with the high degree of automation (in particular regarding warning message generation and dissemination) and with the large amount of functionality caused acceptance and appreciation. However, doubts about manageability were raised. With an increasing amount of components and their functionalities, the complexity of the deployment rises and the knowledge for installation, administration and maintenance is of utmost importance. The components were designed to be used in a professionallyoperated early warning centre which includes regular training and education for the technical staff.

\section{CONCLUDING REMARKS AND OUTLOOK}

The presented work realizes a two-staged CAP generation processes within Crisis Information Management Systems. While threat-centric CAP messages are released by the decision support system (DSS) CCUI, user-tailored warning messages are generated by the ILC. This split significantly unburdens the CCUI of heavy message generation processes and enables a clear separation of concerns that shifts all userspecific message enrichment from the CCUI to the ILC. The CCUI itself has been developed as a modular eclipse RCP application that can be easily extended by hazard- or projectspecific plug-ins, providing new functionalities or GUI components.

Future research will focus on the coupling of the system with dissemination channels that provide broadcast functionality. Although the communication of area geometry has been incorporated, it could not be tested within the project. Such channels will disseminate their warnings (e.g. by text message) to all recipients in range, without considering user preferences such as language. This behaviour foils the efforts spent on generating user-tailored warning messages, but it is often the only way to reach all parts of the population. Whilst the primary focus of the overall project was on the development of a tsunami EWS for the Indian Ocean, the components have been designed and implemented to serve as reference architecture for EWS in general, independent of hazard type and region. The generic nature of the ILC leverages this approach. Other hazard types can be addressed within the ILC by defining new message types and new mes- 
sage templates. Adaptions can be made by providing new plug-ins to the CCUI to integrate hazard or sensor-specific semantics. Analogously, new dissemination channels can be operated by giving adapters to the IDC. Future improvements of the CCUI will also include the option to attach additional resources such as emergency plans or evacuation routes. Moreover, both CCUI and ILC will be upgraded to the new CAP standard v1.2 even if only minor changes were performed. Above all, the transformation of the DEWS project into a set of open source components maintained by an active community has to be performed to leverage a broader usage and further enhancements.

Besides demonstrating the DEWS as a national EWS, the system was also demonstrated as a Wide Area Centre (WAC) serving as a communication network in between national EWS. DEWS followed IOC guidelines such as the communication of thread zones, which has been accomplished by communicating affected areas (coastal forecast zones) via CAP using geocodes. Further efforts will be spent on this type of centre-to-centre communication in the TRIDEC project, which aims among other things for the development of a demonstrator serving in the IOC North East Atlantic and Mediterranean Tsunami EWS (NEAMTWS) network.

\section{ACKNOWLEDGMENTS}

The work presented in this paper is part of the DEWS project funded by the $6^{\text {th }}$ Framework Programme of the European Commission. Further developments will be carried out within the TRIDEC project, funded by the $7^{\text {th }}$ Framework Programme of the European Commission.

\section{REFERENCES}

[1] United Nations International Strategy for Disaster Reduction (UNISDR), "Developing Early Warning Systems: A Checklist", In: Proc of the Third International Conference on Early Warning, 2006, Available from: http://www.unisdr.org/2006/ppew/inforesources/ewc3/checklist/English.pdf [Accessed July 2.2011]

[2] W. Setten, "The Chorist Warning System (CHOR-WARN) Communication to Citizen, in case of an emergency", In: Proc of the DEWS Midterm Conference, 2008, pp. 91-95

[3] S. Jaksch, S. Pfennigschmidt, K. Sandkuhl, and C. Thiel, "Information Logistic Applications for Information-on-Demand Scenarios: Concepts and Experiences from WIND Project", In: Proc of the 29th Euromicro Conference, 2003, p. 141

[4] Working Group on Natural Disaster Information Systems - Subcommittee on Natural Disaster Reduction, "Effective Disaster Warnings", National Science and Technology Council Committee on Environment and Natural Resources, Washington, 2000

[5] E. Berger, G. Peinel, and T. Rose "ERMA Electronic Risk Management Architectures for Small and Medium-sized Communities", Proceedings of the IRMA International Conference, 2007, Available from: http://www.erma-project.org/pdf/ERMA_IRMA2007_paper_final.pdf [Accessed July 2.2011]

[6] U. Raape, T. Steinmetz, M. Wnuk, S. Tessmann, M. Hunold, T. Kukofka, C. Strobl, E. Mikusch, T. Riedlinger, S. Dech, and J. Polleit, "Standardisation of tsunami warning message generation in Indonesia: Approach and implementation", In: Proc of the DEWS Midterm Conference, 2009 ,pp. 111-122

[7] M. Erdik, H. Alcik, O. Ozel, A. Mert, N. Kafadar, B. Tahtasioglu, and A. Korkmaz, "Istanbul Earthquake Rapid Response and the Early Warning System", Geotechnologien Science Report, no 15, pp. 17-25, 2009

[8] K. Thuro, T. Wunderlich, O. Heunecke, J. Singer, P. Wasmeier, S. Schuhbäck, J. Festl, and J. Glabsch, "alpEWEAS - The Aggenalm Landslide - Innovative Developments for an Effective Geo Sensor
Network for Landslide Monitoring and Early Warning", Geotechnologien Science Report, No 13, pp. 33-48, 2009

[9] R. Ackoff, "From Data to Wisdom", J. Appl. Syst. Anal., no. 16, pp. 3-9, 1989

[10] M. K. Lindell, and R. W. Perry, "Behavioral Foundations of Community Emergency Planning”. Washington, D.C.: Hemisphere Publishing Corporation, 2002.

[11] M. K. Lindell, and R. W. Perry, "Communicating Environmental Risk in Multiethnic Communities", Thousand Oaks, California: Sage Publications, Inc.; 2004

[12] T. Terpstra, and H. Vreugdenhil, "Filling in the blanks: Constructing effective flood warning messages using the Flood Warning Communicator (FWC)", In: Proc of the $8^{\text {th }}$ International ISCRAM Conference, Lisbon, 2011, Available from: http://www.iscramlive.org/ISCRAM2011/proceedings/papers/184.pdf [Accessed July 2. 2011]

[13] T. M. Fernandez-Steeger, C. Arnhardt, K. Walter, S. E. Haß, F. Niemeyer, B. Nakaten, S. D. Homfeld, K. Asch, R. Azam, R. Bill, and H. Ritter, "SLEWS - A prototype system for flexible real time monitoring of landslides using an open spatial data infrastructure and wireless sensor networks", Geotechnologien Science. Report., No 13, pp. 3-15, 2009

[14] E. Mutafungwa, and J. Hämäläinen, "Leveraging Femtocells for Dissemination of Early Warning Messages", In: Proc of the DEWS Midterm Conference, 2009, pp. 135-141

[15] A. Botterell, and R. Addams-Moring, "Public warning in the networked age: open standards to the rescue", Commun. ACM, vol. 50, no. 3, pp. 59-60, 2007

[16] Organization for the Advancement of Structured Information Standards (OASIS), "Common Alerting Protocol v. 1.2 Specification", 2010, Available from: docs.oasis-open.org/emergency/cap/v1.2/CAP-v1.2.pdf [Accessed July 2.2011]

[17] ITU - International Telecommunications Union, "Series X: Data Networks, Open System Communications and Security", X.1303, 2007

[18] D. Bunker, and S. Smith "Disaster Management and Community Warning Systems: Inter-Organizational Collaboration and ICT Innovation", In: Proc of the Pacific Asia Conference on Information Systems (PACIS), Hyderabad, India, 2009 Available from: http://aisel.aisnet.org/pacis2009/36/ [Accessed July 2 2011]

[19] R. Iannella, "Incident Notification: Requirements and Frameworks", Technical Report of National ICT Australia (NICTA), ID: PA005037, Australia, 2005, Available from: http://www.nicta.com.au/_data/assets/pdf_file/0020/7256/SafeGu ard2005-Paper.pdf [Accessed July 2:2011]

[20] N. Waidyanatha, G. Gow, and P. Anderson, "Hazard Warnings in Sri Lanka: Challenges of Internetworking with Common Alerting Protocol", In: Proc of the $4^{\text {th }}$ International ISCRAM Conference, Delft, 2007

[21] A. Botterell, "The Common Alerting Protocol: An Open Standard for Alerting, Warning and Notification", In: Proc of the $3^{\text {rd }}$ International ISCRAM Conference, Netwark, NJ, 2006

[22] E. Rohn, "A Survey of Schema Standards and Portals for Emergency Management and Collaboration", In: In: Proc of the $4^{\text {th }}$ International ISCRAM Conference, Delft, 2007

[23] Third Generation Partnership Project (3GPP), "Technical Standard 23.032: Universal Geographical Area Description”, 2006, Available from: http://www.ttc.or.jp/jp/document_list/sum/sumE_JP3GA-23.032(R99)v2.pdf [Accessed July 2. 2011]

[24] International Organization for Standardization (ISO), "Standard 639-1:2002 - Codes for the representation of names of languages - Part 1: Alpha-2 code", 2002, Available from: http://en.wikipedia.org/wiki/ISO_639-1 [Accessed July 2'2011]

[25] J. Wächter, M. Lendholt, and M. Hammitzsch, "Introduction to the Distant Early Warning System (DEWS)", In: Proc of the DEWS Midterm Conference, 2009, pp. 13-18

[26] Organization for the Advancement of Structured Information Standards (OASIS), "Emergency Data Exchange Language (EDXL) Distribution Element (-DE) v.1.0 Specification", 2006

[27] E. Gamma, R. Helm, R. Johnson, and J. Vlissides, "Design Patterns: Elements of Reusable Object-Oriented Software", AddisonWesley Professional, USA, 1995

[28] J. McAffer, J.-M. Lemieux, C Aniszczyk, "Eclipse Rich Client Platform: Designing, Coding, and Packaging Java Applications", Second Edition, Addison-Wesley Professional, USA, 2010 
[29] J. Behrens, A. Androsov, A. Y. Babeyko, S. Harig, F. Klaschka, L. Mentrup, "A new multi-sensor approach to simulation assisted tsunami early warning", Nat. Hazards Earth Syst., vol. 10, pp. 10851100, 2010.

[30] United Nations Educational Scientific and Cultural Organization (UNESCO), "Indian Ocean Tsunami Warning and Mitigation System (IOTWS): Implementation Plan for Regional Tsunami Watch Providers", IOC Information Series, no 8, 2008

[31] M. Lendholt, "Tailoring spatial reference in early warning systems to administrative units", Earth. Sci. Inform., vol. 4, no. 1, pp. 7-16, Springer, 2011
[32] M. Hammitzsch, and M. Lendholt, "Evolving an information logistics database for geospatial early warning systems", Geomatics, Nat. Hazards Risks, vol 2, pp. 95-109, 2011

[33] M. Hammitzsch, M. Lendholt, and M. Á. Esbri, "User interface prototype for gepspatial early warning systems - a tsunami showcase", Nat. Hazards Earth Syst. Sci., 2012 [accepted].

[34] Department of Homeland Security, "Homeland security presidential directive-3: Homeland security advisory system", 2002, Available from: http://www.dhs.gov/xabout/laws/gc_1214508631313.shtm [Accessed 2 July 2011].

(C) Lendholt and Hammitzsch; Licensee Bentham Open.

This is an open access article licensed under the terms of the Creative Commons Attribution Non-Commercial License (http://creativecommons.org/licenses/by-nc/3.0/g) which permits unrestricted, non-commercial use, distribution and reproduction in any medium, provided the work is properly cited. 